\title{
L'expérimentation médicale et les causes de la réticence à la vaccination contre la COVID-19 chez les peuples autochtones du Canada
}

— Citation : CMAJ 2021 March 15;193:E381-3. doi : 10.1503/cmaj.210112-f; diffusion hâtive le 24 février 2021

Voir la version anglaise de l'article ici : www.cmaj.ca/lookup/doi/10.1503/cmaj.210112

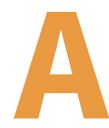

lors que la deuxième vague de la pandémie entraîne une hausse des cas à des niveaux dangereux partout au pays, on constate que les populations autochtones sont particulièrement vulnérables à la maladie à coronavirus 2019 (COVID-19). Les chiffres publiés par l'équipe de coordination de la réponse à la pandémie de COVID-19 chez les Premières Nations du Manitoba sont un bon exemple de cette vulnérabilité. En effet, les membres des Premières Nations représentent à peine plus de $10 \%$ de la population totale du Manitoba, mais comptent pour $71 \%$ des cas actifs de COVID-19 et $50 \%$ des patients aux soins intensifs. Par ailleurs, l'âge médian des personnes décédées de la maladie est de 66 ans chez les Autochtones, contre 83 ans pour l'ensemble de la population de la province ${ }^{1}$.

La situation n'est pas surprenante pour quiconque a lu les dizaines d'études, de rapports et les conclusions des commissions d'enquête parlementaire publiés au cours des 20 dernières années. Étude après étude, la vulnérabilité des communautés des Premières Nations, des Métis et des Inuits face aux crises sanitaires comme celle que nous vivons est manifeste. Cette vulnérabilité découle en grande partie du régime colonial canadien, qui a entravé l'accès des peuples autochtones à des soins de santé adéquats, à une alimentation saine et à l'eau potable, tout en les laissant particulièrement exposés à des conditions de surpeuplement des logements, d'itinérance et d'incarcération.

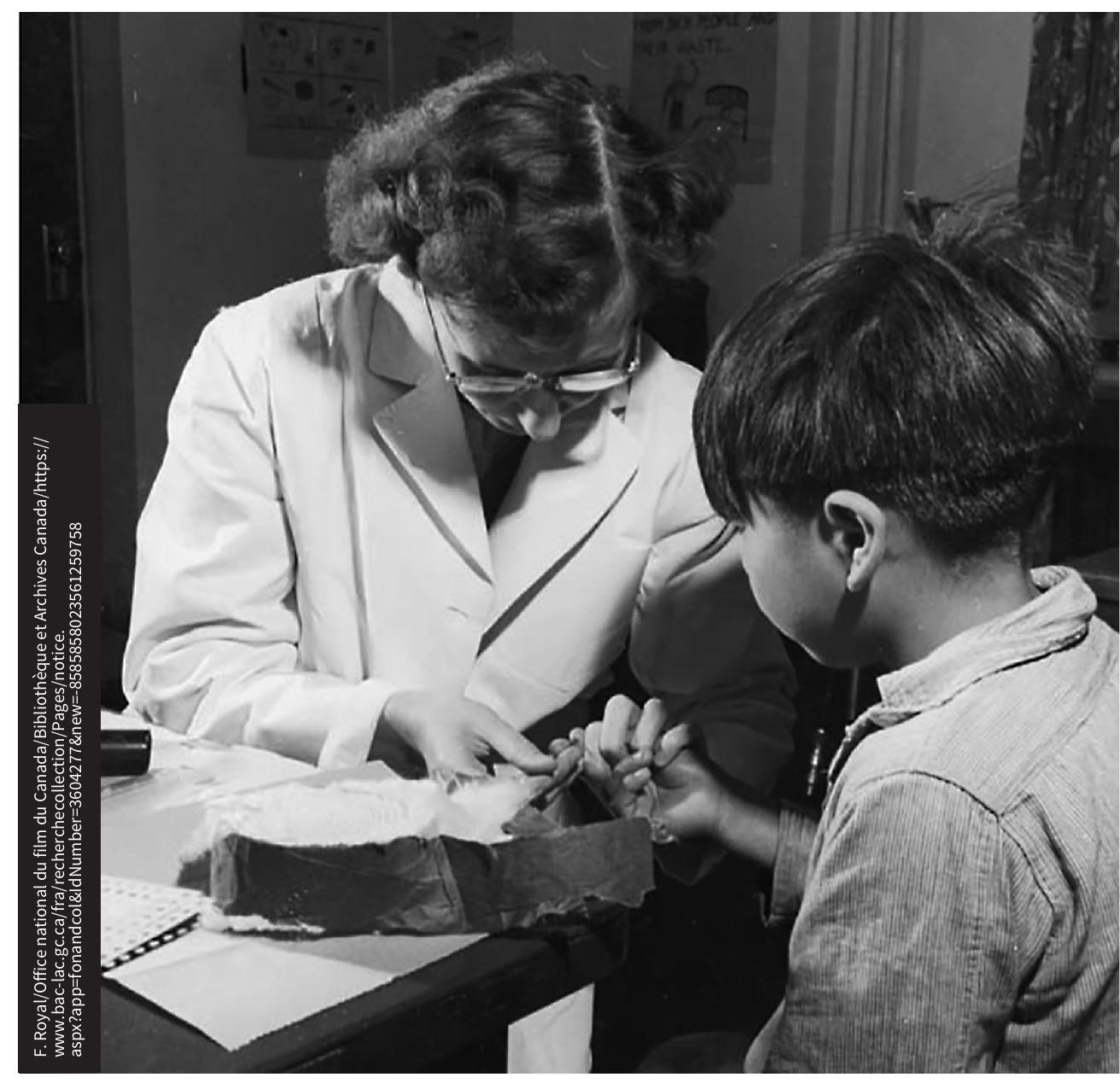

Une infirmière prélève du sang à un garçon au pensionnat indien de Port Alberni, en Colombie-Britannique, lors d'une série d'expériences nutritionnelles menées dans les pensionnats entre 1948 et 1952.

Tous ces facteurs font augmenter le risque de contracter la COVID-19 et de développer de graves complications. II s'avère donc impératif de vacciner en priorité les peuples autochtones contre le coronavirus du syndrome respiratoire aigu sévère 2 (SRAS-CoV-2).

Toutefois, une proportion importante de la population autochtone a exprimé une grande réticence, voire une opposition, 
à la vaccination contre la COVID-19. Après la livraison de 1200 doses du vaccin de Moderna dans sa communauté natale, l'ancien Chef national de l'Assemblée des Premières Nations Matthew Coon Come, a exprimé ses inquiétudes. Dans un message largement diffusé sur les médias sociaux, il a écrit que la population de Mistissini servait maintenant de rats de laboratoire pour ce vaccin expérimental ${ }^{2}$.

\section{Le lourd héritage du passé}

Les craintes et les hésitations formulées par Coon Come ne sont bien sûr pas universelles; en effet, de nombreux dirigeants autochtones ont mentionné explicitement qu'ils étaient très favorables aux vaccins. Néanmoins, il appert que ces préoccupations sont partagées par de nombreux membres des Premières Nations, Inuits et Métis. Comme Sheila North, ancienne grande chef du regroupement autochtone Manitoba Keewatinowi Okimakanak l'explique: "Les gens qui ont connu l'époque des pensionnats, et qui sont maintenant des aînés, se souviennent d'avoir servi de cobayes ou d'avoir servi à tester des vaccins lorsqu'ils étaient enfants, sans leur permission ni celle de leur famille ${ }^{3}$.

Ces préoccupations, craintes et expériences doivent être prises au sérieux par les médecins et les autres professionnels de la santé. Il faut les distinguer des mouvements " anti-vaccination » qui ont pris de l'ampleur sur les médias sociaux ces dernières années. Des populations autochtones ont réellement été soumises à des expériences médicales. L'un des auteurs du présent article a publié des recherches concernant une série d'expériences nutritionnelles menées dans plusieurs communautés cries du Manitoba au cours des années 1940, ainsi que dans 6 pensionnats entre 1948 et $1952^{4}$. L'historienne Maureen Lux a également décrit de nombreuses expériences médicales réalisées sur des peuples autochtones, notamment l'essai du vaccin bilié de Calmette-Guérin, un vaccin expérimental contre la tuberculose, sur des bébés cris et assiniboines en Saskatchewan. Cet essai s'est échelonné sur 12 ans au cours des années 1930 et $1940^{5}$. Une multitude de traitements chirurgicaux et médicamenteux expérimentaux ont également été effectués sans consentement sur des patients autochtones dans les hôpitaux indiens, des établissements de santé ségrégués, au cours des premières années d'après-guerre au Canada ${ }^{5}$.

Le paternalisme raciste a rendu les populations autochtones particulièrement vulnérables aux expérimentations et aux abus dans le domaine médical. L'histoire de Brian Sinclair, mort dernièrement à cause d'un traitement raciste infligé par le personnel hospitalier et les médecins, fait la preuve de la persistance de cet héritage. On l'observe également dans les témoignages des près de 60 femmes autochtones qui ont intenté un recours collectif pour ce qu'elles décrivent comme des stérilisations forcées par des médecins de la Saskatchewan, qui auraient eu lieu au cours des 25 dernières années ${ }^{6}$.

Les peuples autochtones ont donc de très bonnes raisons de se méfier du système médical canadien. Des études récentes montrent que les AfroAméricains, en particulier, sont beaucoup plus susceptibles de refuser la vaccination contre le SRAS-CoV-2 que leurs compatriotes blancs - essentiellement en raison d'expériences bien documentées d'expérimentations et d'abus médicaux. Il faut sans tarder prendre cette question très au sérieux ${ }^{7}$.

\section{La réticence à la vaccination}

La pandémie du virus de la grippe $A$ (H1N1) est un exemple révélateur de la réticence à la vaccination chez les peuples autochtones. Comme le démontrent les études, l'expérience du colonialisme canadien par les peuples autochtones avait " profondément altéré leurs perceptions du vaccin [anti-H1N1] et de la pandémie » et a accru leur hésitation face au vaccin ${ }^{8}$, sentiments que des messages et des interventions problématiques en matière de santé publique ont ensuite exacerbés.

En 2009, durant une éclosion de grippe $\mathrm{H} 1 \mathrm{~N} 1$, un cas troublant est venu renforcer les appréhensions : au lieu d'envoyer des antiviraux, du désinfectant pour les mains et des trousses médicales contre la grippe, le gouvernement fédéral a fait parvenir des housses mortuaires à 4 communautés des Premières Nations du Manitoba ${ }^{9}$. La nouvelle s'est répandue, engendrant de la méfiance partout au pays. Les peuples autochtones ne présentent pas de prédisposition génétique pour la grippe H1N1 ni de risque supplémentaire de complications graves s'ils l'attrapent. Ils ont néanmoins été répertoriés comme un groupe à vacciner contre cette maladie, parmi tous les groupes jugés à risque élevé. Puisque le caractère " autochtone » était la seule caractéristique ciblée, de nombreuses personnes se sont senties comme des cobayes ${ }^{9}$.

Le Canada se prépare déjà à des échecs similaires avec les vaccins contre le SRAS-CoV-2. L'information limitée et tardive sur la COVID-19 et les vaccins approuvés a contribué à la réticence des peuples autochtones à se faire vacciner ${ }^{10}$. Du même coup, on demande aux gens sur le terrain de combler un vide informationnel au sein d'une population déjà sceptique disposant de peu de ressources. Ce vide a poussé Lesa Semmler, membre de l'Assemblée législative d'Inuvik et ancienne infirmière, à faire pression pour que les efforts d'éducation se fassent pour et par la communauté. "Les gens ont besoin de temps, a-t-elle déclaré à la CBC. L'éducation sanitaire doit venir avant [l'arrivée des vaccins $]^{10}$ ».

Comme pour la pandémie de grippe $\mathrm{H} 1 \mathrm{~N} 1$, la priorité accordée aux peuples et aux communautés autochtones dans la campagne de vaccination contre le SRAS-CoV-2 suscite des interrogations. Les récentes déclarations selon lesquelles le Canada ne pourrait pas tenir sa promesse de mettre fin aux avis d'ébullition de l'eau d'ici mars 2021 à cause de la COVID-19 - alors qu'on livre des doses de vaccins de manière prioritaire aux communautés autochtones ne font qu'alimenter l'idée que les peuples autochtones servent à évaluer l'innocuité et l'efficacité du vaccin avant son administration au reste de la population.

\section{Conclusion}

Que convient-il donc de faire pour que le plus grand nombre possible 
d'Autochtones soient vaccinés contre le SRAS-CoV-2, une mesure qui, selon nous, sauvera des vies?

Pour commencer, les médecins et les autres professionnels de la santé doivent se renseigner avant de se rendre dans les communautés pour les vacciner. Un trop grand nombre ignorent l'histoire honteuse du Canada en matière de ségrégation dans les soins de santé et d'expérimentation médicale. Ils comprennent donc mal les racines de la réticence à la vaccination.

Les messages de santé publique sur les risques de l'infection par le SRASCoV-2 et les avantages de la vaccination doivent clairement évoquer l'expérience historique et contemporaine du colonialisme canadien vécue par les peuples autochtones. De plus, il faut décrire les facteurs de risque séparément, plutôt que simplement considérer l'ensemble des Autochtones comme un groupe à risque.

Pour y arriver, il faut renoncer à l'approche universelle en matière de messages de santé publique. Les messages relatifs à la pandémie seront mieux compris s'ils sont transmis directement par des aînés, des dirigeants et des professionnels de la santé autochtones qui jouissent de la confiance de la population et d'une crédibilité à ses yeux. Pour de nombreuses communautés, les messages de santé publique doivent donc être axés non seulement sur la santé et le bienêtre des personnes vaccinées, mais aussi sur la santé et le bien-être des familles, de la communauté, du territoire et des 7 prochaines générations.

À court terme, l'un des problèmes potentiellement insurmontables auquel nous sommes confrontés est la nonreconnaissance d'une responsabilité pour les expériences médicales et autres abus visant les peuples autochtones au sein des établissements de santé canadiens. À long terme, le problème exigera une enquête sur l'histoire de l'expérimentation médicale au Canada ainsi que des réparations aux communautés touchées.

Or, avant d'y parvenir, il faut mettre en œuvre une stratégie de vaccination qui impose aux autorités de prouver aux communautés autochtones que le vaccin est sûr, efficace et sert leurs intérêts. C'est une tâche à laquelle il aurait fallu s'atteler il y a plusieurs mois déjà.

\section{Ian Mosby PhD}

Département d'histoire de l'Université Ryerson, Toronto, Ont.

\section{Jaris Swidrovich PharmD}

École de pharmacie et de nutrition de

l'Université de la Saskatchewan,

Saskatoon, Sask.

\section{Références}

1. Manitoba First Nations COVID-19 Pandemic Response Coordination Team Weekly Bulletin. Winnipeg: Assembly of Manitoba Chiefs; 2021. Accessible ici : https://d5d8ad59-8391-4802-9f0a -f5f5d600d7e9.filesusr.com/ugd/38252a_791803 6c62914724a5b6b3afa3231479.pdf?index=true (consulté le 13 janv. 2021).

2. Pashagumskum J. Former AFN national chief and Cree grand chief speaks out against vaccine. APTN News [Winnipeg] 2020 Jan. 7. Accessible ici : www.aptnnews.ca/national-news/former -afn-national-chief-and-cree-grand-chief-speaks -out-against-vaccine/ (consulté le 13 janv. 2021).

3. Rodriguez J. To tackle vaccine hesitancy, Canada can't ignore race, racism: health experts. CTV News: Coronavirus 2020 Dec. 16, updated 2020 Dec. 17. Accessible ici : www.ctvnews.ca/ health/coronavirus/to-tackle-vaccine-hesitancy -canada-can-t-ignore-race-racism-health-experts -1.5234212 (consulté le 13 janv. 2021).
4. Mosby I. Administering colonial science: nutrition research and human biomedical experimentation in Aboriginal communities and residential schools, 1942-1952. Soc Hist 2013;46:145-72.

5. Lux MK. Separate beds: a history of Indian hospitals in Canada, 1920s-1980s. Toronto: University of Toronto Press; 2016.

6. Indigenous women kept from seeing their newborn babies until agreeing to sterilization, says lawyer. CBC Radio 2018 Nov. 13. Accessible ici : www.cbc.ca/radio/thecurrent/the-current-for -november-13-2018-1.4902679/indigenous-women -kept-from-seeing-their-newborn-babies-until -agreeing-to-sterilization-says-lawyer-1.4902693 (consulté le 13 janv. 2021).

7. Callaghan T, Moghtaderi A, Lueck JA, et al. Correlates and disparities of intention to vaccinate against COVID-19. Soc Sci Med 2020 Dec. 23 [cyberpublication avant impression]. doi: 10.1016/j.socscimed.2020.113638.

8. Driedger SM, Maier R, Furgal C, et al. Factors influencing $\mathrm{H} 1 \mathrm{~N} 1$ vaccine behavior among Manitoba Metis in Canada: a qualitative study. BMC Public Health 2015;15:128.

9. Driedger SM, Cooper E, Jardine C, et al. Communicating risk to Aboriginal Peoples: First Nations and Metis responses to H1N1 risk messages. PLoS One 2013;8:e71106.

10. Zingel A. Work with communities to address vaccine hesitancy, say Indigenous leaders. CBC News North 2021 Jan. 8. Accessible ici : www.cbc. ca/news/canada/north/work-with-communities -vaccine-hesitancy-indigenous-leaders-1.5865677 (consulté le 13 janv. 2021).

Cet article a été révisé par des pairs.

Intérêts concurrents : Aucun déclaré.

Propriété intellectuelle du contenu : Il s'agit d'un article en libre accès distribué conformément aux modalités de la licence Creative Commons Attribution (CC BY-NC-ND 4.0), qui permet l'utilisation, la distribution et la reproduction dans tout médium à la condition que la publication originale soit adéquatement citée, que l'utilisation se fasse à des fins non commerciales (c.-à-d., recherche ou éducation) et qu'aucune modification ni adaptation n'y soit apportée. Voir : https://creativecommons.org/ licenses/by-nc-nd/4.0/deed.fr. 